Editorial

\title{
Yeast Biotechnology 3.0
}

\author{
Ronnie G. Willaert ${ }^{1,2} \mathbb{D}$ \\ 1 Research Group Structural Biology Brussels, Alliance Research Group VUB-UGent \\ NanoMicrobiology (NAMI), IJRG VUB-EPFL BioNanotechnology \& NanoMedicine (NANO), \\ Vrije Universiteit Brussel, 1050 Brussels, Belgium; Ronnie.Willaert@vub.be \\ 2 Department of Bioscience Engineering, University Antwerp, 2000 Antwerp, Belgium
}

Received: 23 July 2020; Accepted: 27 July 2020; Published: 29 July 2020

Keywords: Saccharomyces cerevisiae; non-Saccharomyces yeasts; fermentation-derived products; fermented beverages; wine; beer; coffee bean fermentation; flavor; itaconic acid production; bioethanol production; bioreactors; yeast micro- and nanobiotechnology

\section{Yeast Biotechnology 3.0}

This Special Issue is a continuation of the first and second "Yeast Biotechnology" Special Issue series of the journal Fermentation (MDPI). This issue compiles the current state-of-the-art of research and technology in the area of "yeast biotechnology" and highlights prominent current research directions in the fields of yeast micro- and nanobiotechnology, brewer's yeasts and beer fermentation, wine yeasts and wine fermentation, coffee bean fermentation and new developments in biochemicals production by yeasts. We very much hope that you enjoy reading it and are looking forward to the next Special Issue "Yeast Biotechnology 4.0" to appear in 2020-2021 (https://www.mdpi.com/journal/fermentation/special_issues/yeast_4).

\section{Yeast Micro- and Nanobiotechnology}

Living cell microarrays in microfluidic chips allow the non-invasive multiplexed molecular analysis of single cells. Yvanoff et al. [1] developed a simple and affordable perfusion microfluidic chip containing a living yeast cell array composed of a population of green fluorescent protein (GFP)-tagged Saccharomyces cerevisiae clones. Mechanical patterning in microwells and robotic piezoelectric cell dispensing in the microwells were combined to construct the cell arrays. The developed microfluidic technology has the potential to be easily upscaled to a high-density cell array, allowing one to perform dynamic systems biology (proteomics and localisomics) experiments on growing cells.

Yeast resistance to antifungal drugs is a major public health issue. Fungal adhesion onto the host mucosal surface is still a partially unknown phenomenon that is modulated by several actors, among which fibronectin plays an important role. Targeting the yeast adhesion onto the mucosal surface could lead to a highly efficient treatment for Candida infections. A nanoscale approach to study the behavior of the pathogenic yeast $C$. albicans was develop by Kohler et al. [2]. Using atomic force microscopy (AFM)-based detection of the nanoscale motions of the yeast cells, it was demonstrated that strongly adhering strains reduce their nanomotion activity upon fibronectin exposure, whereas low adhering C. albicans remain unaffected. These results open novel avenues to explore cellular reactions upon exposure to stimulating agents and to monitor, in a rapid and simple manner, the adhesive properties of $C$. albicans.

\section{Brewer's Yeasts and Beer Fermentation}

Due to changing lifestyle trends and legislation, there is a growing demand for non-alcoholic beers (NABs). In recent years, production methods have been improved and the use of non-Saccharomyces 
yeasts has been investigated. Non-Saccharomyces yeasts are interesting, since fruity ester aromas can be introduced. Bellut et al. [3] evaluated several Cyberlindnera strains for NAB production. It was demonstrated that the selected Cyberlindnera subsuciens was suitable to produce a fruity NAB. The outcome strengthens the position of non-Saccharomyces yeasts as a serious and applicable alternative to established methods in NAB brewing.

For some years, there has also been a new trend in using non-conventional yeasts to change the aroma profile of traditional beers. Canonico et al. [4] proposed the use of Torulaspora delbrueckii to obtain a beer with a distinctive aromatic taste. S. cerevisiae/T. delbrueckii mixed fermentations resulted in beers with increased concentrations of some aromatic compounds such as ethyl hexanoate, $\alpha$-terpineol, and $\beta$-phenyl ethanol and an emphasized note of fruity/citric and fruity/esters notes.

Brewer's yeast flocculation is a well-appreciated characteristic of industrial brewer's strains, since it allows the removal of the cells from the beer in a cost-efficient way. However, many industrial strains are non-flocculent and genetic interference to increase the flocculation characteristics is not appreciated by the consumers. Optimization of the brewer yeast towards a more flocculating phenotype can lead to a more efficient beer production and a higher final beer quality. An attractive approach to enhance the attributes of microorganisms is the adaptive laboratory evolution (ALE) approach. Kayacan et al. [5] applied ALE to non-flocculent industrial S. cerevisiae brewer's strains using small continuous bioreactors and obtained an aggregative "snowflake" phenotype. It was demonstrated that ALE increased the sedimentation behavior and that no major flavor changes in the produced beer was detected.

\section{Wine Yeasts and Wine Fermentation}

Before cryopreservation was an established method to store wine yeasts, strain collections were stored at room temperature on agar slants in glass reagent tubes covered with vaspar and sealed with cotton plugs. Matti et al. [6] characterized 60 strains from the old wine yeast collection from the Geisenheim Yeast Breading collection and confirmed the suitability of storing yeasts by this old method. White wine fermentations and post-fermentation aroma analyses were performed. It was shown that this old strain collection bears treasures for direct use either in wine fermentations or for incorporation in yeast breeding programs aimed at improving modern wine yeasts.

Yeasts naturally occur in vineyards on the grapes and consequently in wines. Kačániová et al. [7] identified yeasts on 30 grape varieties and 60 wine samples. MALDI-TOF mass spectrometry was used for the identification of yeasts and a total of 1668 isolates were identified. The most isolated species from the grapes was Hanseniaspora uvarum, and from wine, it was S. cerevisiae.

The selection of the yeast(s) is one of the most important "tools" for modulating flavor and color in wines. Therefore, Vilela [8] reviewed the role of Saccharomyces and non-Saccharomyces yeasts, as well as lactic acid bacteria, on the perceived flavor and color of wines and the choice that winemakers can make by choosing to perform co-inoculation or sequential inoculation. This choice will help them to achieve the best performance in enhancing these wine sensory qualities, avoiding spoilage and the production of defective flavor or color compounds.

During the fermentation of wine, the malolactic fermentation (MLF), which is performed by lactic acid bacteria, takes a prominent role, since it influences the wine flavor and its microbiological stability. Izquierdo-Cañas et al. [9] studied the effects of simultaneous inoculation of a selected S. cerevisiae yeast strain with two different commercial strains of wine bacteria Oenococcus oeni at the beginning of the alcoholic fermentation on the kinetics of the MLF, wine chemical composition, and organoleptic characteristics in comparison with spontaneous MLF in Tempranillo grape must. It was shown that co-inoculation reduced the overall fermentation time by up to 2 weeks, resulting in a reduced volatile acidity. The fermentation-derived wine volatiles profile was distinct between the co-inoculated wines and spontaneous MLF and was influenced by the selected wine bacteria. Co-inoculation resulted in wines with very little lactic acid and buttery flavors. 
Icewine is a sweet dessert wine that is fermented from the juice of naturally frozen grapes. The high concentration of sugars in Icewine juice results in considerable osmotic stress in the fermenting S. cerevisiae. Yeast can combat this stress by increasing the internal concentration of glycerol by activating the high osmolarity of the glycerol response to synthesize glycerol and by actively transporting glycerol into the cell from the environment. Muyssen et al. [10] investigated the role of the glycerol/ $\mathrm{H}^{+}$symporter Stl1p in Icewine fermentations. Therefore, a strain of the common Icewine yeast S. cerevisiae K1-V1116 that lacks STL1 was constructed using a developed CRISPR-Cas9-based genome editing method. The results demonstrate that glycerol uptake by Stl1p has a significant role during osmotically challenging Icewine fermentations, despite potential glucose downregulation.

There is a high interest in monitoring the changes in biochemical compounds that are changed during the alcoholic wine fermentation, since the management of the alcoholic fermentation is crucial in shaping the wine quality. Berbegal et al. [11] demonstrated the use of proton-transfer reaction-mass spectrometry coupled to a time-of-flight mass analyzer (PTR-ToF-MS) to monitor on-line volatile organic compounds (VOCs). The effect of multiple combinations of two Saccharomyces strains and two non-Saccharomyces strains (Metschnikowia pulcherrima and Torulaspora delbrueckii) on the content of VOCs in wine was assessed.

\section{Coffee Bean Fermentation}

Yeast fermentation of coffee beans improves the functionality of the beans and the quality of the coffee. Haile and Kang [12] evaluated the effect of green coffee bean fermentation with Wickerhamomyces anomalu. They demonstrated an improved functionality of the coffee beans, which was reflected into an increased total phenol and total flavoid content and a reduced total tannin content, and an improvement of the 2,2-diphenyl-1-picrylhydrazyl radical scavenging assay and the ferric reducing antioxidant power. Significant differences were also found in the superoxide dismutase activity.

The quality of coffee can also be improved by fermenting the mucilage layer of the coffee mixture with lactic acid bacteria and yeasts. da Silva Vale et al. [13] studied the effect of co-inoculation of Pichia fermentans and Pediococcus acidilactici on metabolite production during fermentation and the volatile composition of the coffee beans. They demonstrated an improved fermentation efficiency and a positive influence on the chemical composition of the coffee beans

\section{New Developments in Biochemicals Production}

Over the last few years, intense research has been focused on the generation of alternative renewable biofuels by fermenting agriculture waste using yeasts. One of the trends is the production of bioethanol by the fermentation of cellulosic and hemicellulosic biomass. The fermentation and assimilation of xylose (the second most abundant hemicellulosic carbohydrate) is still a bottleneck in the efficient production of bioethanol, since the conventional yeast $S$. cerevisiae cannot consume xylose. However, non-conventional yeasts, such as Spathaspora passalidarum and Pichia stipitis, can utilize xylose. Selim et al. [14] reviewed recent advances in xylose metabolizing yeasts, with special emphasis on S. passalidarum for improving bioethanol production.

Itaconic acid is an interesting biochemical for the polymer industry, since it can be produced from renewable substrates (such as lignocellulosic based hydrolysates) by fermentation and can replace petrochemical-based chemicals. It can be produced by the filamentous fungus Aspergillus terreus. Recently, alternative itaconic acid-producing yeasts such as the basidiomycetous yeasts of the family Ustilaginaceae, have been studied. Krull et al. [15] evaluated Ustilago rabenhorstiana as an alternative natural itaconic acid producer. By the optimization of media components and process parameters, a final itaconic acid concentration of $50 \mathrm{~g} \mathrm{~L}^{-1}$ using fed-batch fermentation was obtained. Moreover, itaconic acid was produced from different sugar monomers based on renewable feedstocks and the robustness against weak acids as sugar degradation products was confirmed. 
Acknowledgments: The Belgian Federal Science Policy Office (Belspo) and the European Space Agency (ESA) PRODEX program supported this work. The Research Council of the Vrije Universiteit Brussel (Belgium) and the University of Ghent (Belgium) are acknowledged to support the Alliance Research Group VUB-UGhent NanoMicrobiology (NAMI), and the International Joint Research Group (IJRG) VUB-EPFL BioNanotechnology and NanoMedicine (NANO).

Conflicts of Interest: The author declares no conflict of interest.

\section{References}

1. Yvanoff, C.; Torino, S.; Willaert, R.G. Robotic Cell Printing for Constructing Living Yeast Cell Microarrays in Microfluidic Chips. Fermentation 2020, 6, 26. [CrossRef]

2. Kohler, A.-C.; Venturelli, L.; Kannan, A.; Sanglard, D.; Dietler, G.; Willaert, R.; Kasas, S. Yeast Nanometric Scale Oscillations Highlights Fibronectin Induced Changes in C. albicans. Fermentation 2020, 6, 28. [CrossRef]

3. Bellut, K.; Michel, M.; Zarnkow, M.; Hutzler, M.; Jacob, F.; Atzler, J.J.; Hoehnel, A.; Lynch, K.M.; Arendt, E.K. Screening and Application of Cyberlindnera Yeasts to Produce a Fruity, Non-Alcoholic Beer. Fermentation 2019, 5, 103. [CrossRef]

4. Canonico, L.; Ciani, E.; Galli, E.; Comitini, F.; Ciani, M. Evolution of Aromatic Profile of Torulaspora delbrueckii Mixed Fermentation at Microbrewery Plant. Fermentation 2020, 6, 7. [CrossRef]

5. Kayacan, Y.; Van Mieghem, T.; Delvaux, F.; Delvaux, F.R.; Willaert, R. Adaptive Evolution of Industrial Brewer's Yeast Strains towards a Snowflake Phenotype. Fermentation 2020, 6, 20. [CrossRef]

6. Matti, K.; Bernardi, B.; Brezina, S.; Semmler, H.; von Wallbrunn, C.; Rauhut, D.; Wendland, J. Characterization of Old Wine Yeasts Kept for Decades under a Zero-Emission Maintenance Regime. Fermentation 2020, 6, 9. [CrossRef]

7. Kačániová, M.; Kunová, S.; Sabo, J.; Ivanišová, E.; Žiarovská, J.; Felsöciová, S.; Terentjeva, M. Identification of Yeasts with Mass Spectrometry during Wine Production. Fermentation 2020, 6, 5. [CrossRef]

8. Vilela, A. Modulating Wine Pleasantness Throughout Wine-Yeast Co-Inoculation or Sequential Inoculation. Fermentation 2020, 6, 22. [CrossRef]

9. Izquierdo-Cañas, P.M.; Ríos-Carrasco, M.; García-Romero, E.; Mena-Morales, A.; Heras-Manso, J.M.; Cordero-Bueso, G. Co-Existence of Inoculated Yeast and Lactic Acid Bacteria and Their Impact on the Aroma Profile and Sensory Traits of Tempranillo Red Wine. Fermentation 2020, 6, 17. [CrossRef]

10. Muysson, J.; Miller, L.; Allie, R.; Inglis, D.L. The Use of CRISPR-Cas9 Genome Editing to Determine the Importance of Glycerol Uptake in Wine Yeast During Icewine Fermentation. Fermentation 2019, 5, 93. [CrossRef]

11. Berbegal, C.; Khomenko, I.; Russo, P.; Spano, G.; Fragasso, M.; Biasioli, F.; Capozzi, V. PTR-ToF-MS for the Online Monitoring of Alcoholic Fermentation in Wine: Assessment of VOCs Variability Associated with Different Combinations of Saccharomyces/Non-Saccharomyces as a Case-Study. Fermentation 2020, 6, 55. [CrossRef]

12. Haile, M.; Kang, W.H. Antioxidant Properties of Fermented Green Coffee Beans with Wickerhamomyces anomalus (Strain KNU18Y3). Fermentation 2020, 6, 18. [CrossRef]

13. da Silva Vale, A.; de Melo Pereira, G.V.; de Carvalho Neto, D.P.; Rodrigues, C.; Pagnoncelli, M.G.B.; Soccol, C.R. Effect of Co-Inoculation with Pichia fermentans and Pediococcus acidilactici on Metabolite Produced During Fermentation and Volatile Composition of Coffee Beans. Fermentation 2019, 5, 67. [CrossRef]

14. Selim, K.A.; Easa, S.M.; El-Diwany, A.I. The Xylose Metabolizing Yeast Spathaspora passalidarum is a Promising Genetic Treasure for Improving Bioethanol Production. Fermentation 2020, 6, 33. [CrossRef]

15. Krull, S.; Lünsmann, M.; Prüße, U.; Kuenz, A. Ustilago Rabenhorstiana-An Alternative Natural Itaconic Acid Producer. Fermentation 2020, 6, 4. [CrossRef]

(C) 2020 by the author. Licensee MDPI, Basel, Switzerland. This article is an open access article distributed under the terms and conditions of the Creative Commons Attribution (CC BY) license (http://creativecommons.org/licenses/by/4.0/). 